\title{
Brief Strategic Family Therapy
}

\author{
Viviana Horigian ${ }^{1}$ \\ Michael Robbins ${ }^{1}$ \\ José Szapocznik ${ }^{1}$
}

\begin{abstract}
Behavioral problems in adolescence rarely appear in isolation. Most commonly, problems occur in clusters that may include combinations of several problem behaviors, such as truancy, vandalism, gang participation and association with deviant peers, conflict with parents, poor academic performance and attendance, violence, drug use, risky sexual behaviors, and delinquency. The pervasiveness of behavior problems across multiple settings often causes considerable distress to parents, school personnel, and society as a whole.

Adolescents with behavior problems also pose significant challenges for mental health professionals. Most adolescents are referred to treatment by a third party, such as their parents, school, or the court, and they are typically not troubled by their own behavior, which - combined with their general recalcitrance toward authority - make them quite formidable to deal with in the treatment context. The challenge for mental health counselors is further compounded by the fact that the behavior problems often occur in contexts of severe familial and social disruption; problems which most therapists are ill prepared to address, but which we believe are critical components of successful treatment for these youths. For example, clinical theory and research have identified specific family interaction patterns that are linked to adolescent behavior problems. This knowledge has facilitated the development of family-based interventions strategies that specifically target these patterns One intervention that has received considerable support in research studies is Brief Strategic Family Therapy (BSFT), an intervention developed over the past 30 years at the Center for Family Studies of the University of Miami. $\quad B S F T$ is specifically designed to provide families with the tools to overcome adolescent behavior problems and the family dysfunction that often accompanies these adolescent problems through: 1) focused interventions to correct maladaptive patterns of family interaction, and 2) skills building strategies to strengthen families.
\end{abstract}

${ }^{1}$ Center for Family Studies, University of Miami, Miami, Florida, U.S.A. 


\section{Introduction}

Behavioral problems in adolescence rarely appear in isolation. Most commonly, problems occur in clusters that may include combinations of several problem behaviors, such as truancy, vandalism, gang participation and association with deviant peers, conflict with parents, poor academic performance and attendance, violence, drug use, risky sexual behaviors, and delinquency. The pervasiveness of behavior problems across multiple settings often causes considerable distress to parents, school personnel, and society as a whole.

Adolescents with behavior problems also pose significant challenges for mental health professionals. Most adolescents are referred to treatment by a third party, such as their parents, school, or the court, and they are typically not troubled by their own behavior, which - combined with their general recalcitrance toward authority - make them quite formidable to deal with in the treatment context. The challenge for mental health counselors is further compounded by the fact that the behavior problems often occur in contexts of severe familial and social disruption; problems which most therapists are ill prepared to address, but which we believe are critical components of successful treatment for these youths. For example, clinical theory and research have identified specific family interaction patterns that are linked to adolescent behavior problems. This knowledge has facilitated the development of family-based interventions strategies that specifically target these patterns One intervention that has received considerable support in research studies is Brief Strategic Family Therapy (BSFT), an intervention developed over the past 30 years at the Center for Family Studies of the University of Miami. BSFT is specifically designed to provide families with the tools to overcome adolescent behavior problems and the family dysfunction that often accompanies these adolescent problems through: 1) focused interventions to correct maladaptive patterns of family interaction, and 2) skills building strategies to strengthen families.

\section{Common Risk Protective factors in USA Cultural Context}

Children, adolescents, and their families develop, not in isolation from their context, but rather in constant interaction with it. The interface between individuals, families, and social systems create a contextual reality that involves a combination of patterns of relationships that serve to protect or place the child at risk for developing behavior problems. Although this perspective acknowledges that a variety of individual factors (e.g., personal identity) may serve to protect increased risk, family-based interventions focus primarily on family, peer (family-peer), and school (family-school) relationships.

For family-based interventions such as BSFT, the family is the primary context for the child's development and socialization. Adaptive family functioning can help to protect children and adolescents from developing behavior problems. For example, parental investment, open and active parent-child communication and bonding, monitoring and supervision of peer relationships and activities have all been associated with reducing adolescents risk for behavior problems. In contrast, poor parenting, high family conflict, and a lack of monitoring and supervision of adolescent activities have been associated with adolescent behavior problems (Szapocznik \& Coatsworth, 1999). The challenge in BSFT is 
to capitalize on the strengths of families and individual family members to alter the relational context in a manner that reduces risk and increased protection.

Like other family approaches to the treatment of adolescent problem behavior), BSFT also acknowledges the critical influence of other relevant systems that influence children and adolescents - most prominently, the school and peer systems. Adolescents with behavior problems often have considerable difficulties at school. They frequently are suspended or expelledfrom school, and many perform poorly academically. Interactions between the youth and school personnel are hostile, defiant, and there is rejection on both sides. Unfortunately, many parents do not have the skills needed to effectively minimize the damage that is created at school as adolescents display problems at school. In BSFT, a considerable amount of effort is devoted to repairing these relationships and reconnecting the adolescent (and her/his parents) to school. Much of this work involves building parents skills and improving parent-school connections to maximize the parents ability to function as a leader in this aspect of their child's life.

Anotherimportant system for adolescents is the peer network. Research has consistently documented that association with deviant peers might be the most significant predictor of adolescent behavior problems. This link is not surprising given the relational relevance of the peer group during adolescence. In BSFT, considerable attention is devoted to weakening the adolescent's links to deviant peers by improving family relationships, improving parent's ability to monitor and supervise adolescent's activities, and connecting adolescents with pro-social peer networks.

\section{Theory of the Problem: Structural - Systemic}

BSFT is based on the systemic theory of family functioning. The family as a system that influences all its members, has its own unique characteristics and properties that emerge and are apparent only when family members interact. In BSFT, this view of the family system is evident in the following assumptions: 1) The family is a system with interdependent/interrelated parts; 2) The behavior of one family member can only be understood by examining the context (i.e., family) in which it occurs; 3) Interventions must be implemented at the family level and must take into account the complex relationships within the family system; 4) Families are nested within complex social systems; 5) Child symptoms are associated with maladaptive patterns of social interactions with the family / and or between child/family and social ecology. and,

6) Social interactions are defined structurally.

Understanding the structure of the family helps us to explain the mechanisms by which the behaviors of family members are interdependent. In BSFT, the view of structure is evident in the following assumption: Repetitive interactions (i.e., ways family members behave with one another) are either successful or unsuccessful in achieving the goals of the family and/or its individual members.

\section{Structural- Systemic Diagnosis of the Family}

In BSFT, family interactions are assessed along five dimensions: organization, resonance, developmental stage, identified patienthood, and conflict resolution. Accurate diagnosis 
along these dimensions can only be achieved through direct observation of the family. The first goal in the diagnostics process is to create opportunities for family members to interact directly with one another, with minimal intervention from the therapist. BSFT therapists must create a therapeutic context where family members are free to interact in their typical style. These "enactments" permit the therapist to observe directly how the family typically behaves. This structural diagnosis is critical for accurately identifying the family's characteristic patterns of interaction, i.e., the family's structure. The following are patterns of family interactions that often occur in families of problem behavior adolescents, and which when corrected can support correction of the adolescents' problem behaviors.

a) Organization

Organization in a family can be examined through three aspects: Leadership, Subsystem Organization, and Communication Flow.

Leadership is defined as the distribution of authority and responsibility within the family. In functional two-parent families, leadership is in the hands of the parents. In modern societies, both parents and parent figures usually share authority and decisionmaking. Frequently, in one-parent families, the parent shares some of the leadership with an older child. In the case of a single parent living within an extended family framework, leadership may be shared with an uncle, aunt or grandparent. Assessment of Leadership includes evaluating hierarchy, behavior control, and, guidance. The BSFT therapist must identify who is in charge of directing the family, who are the family members in positions of authority, evaluate that leadership is in the appropriate hands, or that is shared between (or among) the appropriate people, that hierarchy is assigned appropriately with respect to age, role and function within the family. Behavior control and guidance are other aspects of the assessment of Leadership. Parent figures should be responsible for maintaining behavior control and for providing guidance, although some of this responsibility can be delegated to other family members. In general, leadership should be with the parental figures, although some leadership can be delegated to older children as long as such delegation is not overly burdensome, is age-appropriate, and is delegated and not usurped.

Families have both formal subsystems (e.g., spouses, siblings, grandparents, etc.) and informal subsystems (e.g., the older women, the people who manage the money, the ones who do the housekeeping, the ones who play chess). Each of these subsystems must have a certain degree of privacy and independence (i.e., boundaries). Assessment of the family's subsystem organization, includes evaluating the adequacy and appropriateness of the subsystems that exist in the family, as well as alliances, triangulation, and subsystem membership. Alliance between parental figures should be strong, whereas problematic alliances tend to occur between adult and child, particularly when a parent is left out of the alliance or when a parent and a child form a coalition against the other parent. Triangulation is illustrated when two parental authority figures have a disagreement and introduce a third, less powerful person to diffuse the conflict rather than resolving the disagreement between themselves. Triangles are maladaptive because they prevent the resolution of a conflict between two authority figures. Unresolved conflict becomes an ongoing source of frustration, irritation, and anger for all family members involved. The triangulated youth typically receives the brunt of much of the unhappiness of both parents and frequently becomes the families "symptom-bearer" or "Identified Patient".

The final category of organization looks at the nature of communication. In functional families, communication flow is characterized by Directness and Specificity. Good 
communication involves each family member communicating directly and clearly to other family members.

Maladaptive family interactions found in families with adolescent behavior problems in the organization dimension include parents being unable to collaborate and maintain behavior control, youth in a powerful role, and one parent and problem youth in coalition.

\section{b) Resonance}

Resonance defines the emotional and psychological accessibility or distance between family members. Assessment of resonance between family members includes evaluation of the permeability of interpersonal boundaries. The BSFT Therapist needs to identify where one person or group of people ends and where the next one(s) begins, and family boundaries, which dictate how family members respond or react emotionally to one another. Family members over-react to one another when they are too engaged and when emotional, psychological, or physical boundaries are excessively close. On the contrary, family members who are not engaged at all do not react to one another; they ignore each other. Enmeshment and disengagement represent the two extremes in the evaluation of this dimension. Family members with the proper (i.e., moderate) degree of engagement react to one another while maintaining their separate individuality. Examples of enmeshment are mind reading, mediated responses (one family member speaks to another family member on behalf of a third family member), simultaneous speeches, interruptions, continuations (one family member completes the statement of another family member), personal control (one family member assumes they are able to control the thoughts, feelings, or behaviors of another family member), physical loss of distance (one family member controls the behaviors of another through physical means), joint affective reactions (family members share the same emotions), engagement reactions (the reactions of one family member trigger the reactions of another). The notion of resonance and permeability of boundaries applies not only to the relationship between family members, but also to the relationship between a particular subsystem (such as the marital couple) and the rest of the family.

Maladaptive patterns observed in families with adolescent behavior problems include: problem youth and one parent over- involved; the other parent under- involved and little intimacy in either relationship.

\section{c) Developmental Stage}

Individuals go through a series of developmental stages, ranging from infancy to childhood, adolescence to young adulthood, and from middle age to old age. Each stage involves different roles and responsibilities. Families are comprised of multiple individuals who are often at different stages of development. It is not always realized, however, that families also go through a series of developmental stages; and, in order for its members to continue to function in a healthy way, family members need to behave in ways that are appropriate at each developmental level. As families grow and develop, changes occur in family composition as well as in the behaviors that family members are able to engage in. Families also must re-organize to undergo major developmental milestones, such as when a child is born, children leave home to live on their own, when a partner retires or when a marital couple separates. Other significant milestones include the death or serious illness of any family member, the break up of parents' marriages, the temporary custody of children by one or another parent, a grandparent, or a foster parent, the reunification of portions of a family - and the many other permutations that families undergo as members are added and 
subtracted. All of these milestones bring stress and require that the family adapt to the new circumstances. Failure to adapt (i.e., establish new interaction patters) may lead to the emergence of adolescent behavior problems.

In our work with adolescents, one of the most common developmental problems for adolescents involves parents continuing to treat an adolescent as if $\mathrm{s} / \mathrm{he}$ were a younger child. Therefore, the family behaviors that were adaptive at one time (when the adolescent was a child) become maladaptive when circumstances change. During adolescence, parents must be able to continue to be involved and monitor their adolescent's life, but now from a distinctly different perspective that allows for increasing autonomy in the youth. The family's flexibility - ability to adapt in general — will have a profound impact on its ability to adapt to new circumstances, including those new circumstances caused by developmental shifts. In general, flexible families are healthier families.

When a family's developmental stage is analyzed, four major sets of tasks and roles are assessed: parenting tasks and roles, marital tasks and roles, sibling tasks and roles, and extended family's tasks and roles. How individuals and the family are functioning in each of these areas is evaluated in reference to what is normative or expected at that stage of individual and familial development. Examples of maladaptive patterns include immature parenting behaviors, children that are treated or act too young and are given few opportunities for responsible behavior, or when the child is overloaded with adult tasks and exhibit parent like behaviors.

\section{d) Identified Patient}

Adolescents with behavior problems often become the repository for all the family's blame, that is, they are the family's "identified patient." This is certainly often the case for problem behavior adolescent who with their problem behaviors attract the family's frustration and anger toward them.

Because the identified patient (IP) is the target of family negativity, it allows the family to perpetuate its rigid patterns of maladaptive interactions. The IP may also receive considerable support from some family members who nurture in the IP inappropriate behaviors; failing to provide needed sanctions or consequences. That is, family interactions tend to revolve around the adolescent. Families of youth who have problems with drug useand present other behavior problems often exhibit a high degree of negativity in the form of blaming, criticizing, accusing, hopelessness, and rejection, mostly toward the IP. More central, rigid and maladaptive family "patienthood" is usually marked by excessive negativity, or, on the other hand, by enabling behaviors such as protection and even nurturance of the symptom. The more rigid the Identified Patienthood, the more that family members blame and centralize the adolescent, the more difficult the family interactions are to change.

\section{e) Conflict Resolution}

While solving differences of opinion is always challenging, it is so much more challenging when it is done in the context of a history of highly conflictive relationships. Disagreements are natural and all families experience them. Conflict resolution style in a family can adopt one of five styles: denial, avoidance, diffusion, conflict emergence without resolution, and conflict emergence with resolution. For a family to function well, it must use the full range of styles in solving conflicts. Different styles may work well at different times. Ideally, a well-functioning family uses all conflict management styles, 
according to the needs of the situation. Emergence of the conflict with a subsequent resolution is generally considered to be the best solution in areas of conflict that are of significant importance to the family's functioning. Separate accounts and opinions regarding a particular conflict are clearly expressed. Then, the family is able to negotiate a solution that is acceptable to all family members involved.

Maladaptive patterns observed in families with adolescent behavior problems, and particularly with the families of Latin American origin with which we work include high conflict level with conflict diffusion characterized by blaming, accusing, or changing topics.

\section{Theory of Change: Strategic Approach}

BSFT is a strategic approach that uses practical, problem-focused, and planned interventions. This strategic approach emerged from an explicit focus on developing an intervention that was quick and effective in eliminating symptoms. In $\boldsymbol{B S F T}$, this strategic approach is evident in the following assumptions:

1) Interventions are practical. That is, interventions are tailored to the unique characteristics of families and are implemented to achieve attainable treatment goals as quickly and effectively as possible. Having identified a problematic interaction, $\boldsymbol{B S F T}$ will proceed to design a specific strategy for change that is based on what will make people behave in ways that produce change. These are strategies that are aimed not at developing insight or awareness, but rather at creating new sequences of behavior that eliminate the dysfunctional or change-resistant behaviors. Strategic interventions are particularly visible in the reframes selected to highlight some aspect of the families interactions that can help the family to move to more supportive interactions. For example, reframing anger for passion and concern.

2) Interventions are problem focused. A problem-focused approach targets only those patterns of interactions that most directly influence the youth's psychosocial adjustment and antisocial behaviors and targets one problem at a time. Thus, not all maladaptive interactions are targeted for change, but only those that are directly linked to the problem behavior. For example, to correct adolescent problem behaviors, parents (or parent figures) must collaborate around parenting. Parents who differ will find that the adolescent takes advantage of their difference to act out. Therefore, when parents are not collaborating, interventions are targeted at improving the collaboration among parents. This is not always done directly but rather in a strategic fashion.

3) Interventions are well planned. The BSFT therapist determines what seem to be the maladaptive interactions (i.e., interactions that are directly related to the youth's behavior problems), determines which of these might be targeted, and establishes a focused plan to help the family develop more effective patterns of interaction. Many families of problem behavior adolescents are overwhelmed by their many problems. It is easy for a therapist to become overwhelmed unless the therapist prioritizes carefully the targets for change and remains focused on their target. 


\section{Theory of Change: Circular Sequence}

In practice, $\boldsymbol{B S F T}$ is delivered as an integrated strategy. This strategy of therapy must be thoughtfully planned, though the actual therapeutic moves must be authentic and flowing from the moment. In general, the strategy of BSFT follows these steps: Joining, Enactment, Interactional Diagnosis, Treatment Plan and finally, Restructuring Change. (See figure 1) Each of these steps is crucial for effective change to occur. Without any of them, therapy with problem behavior adolescents often fails.

Fig 1. BSFT Change Sequence

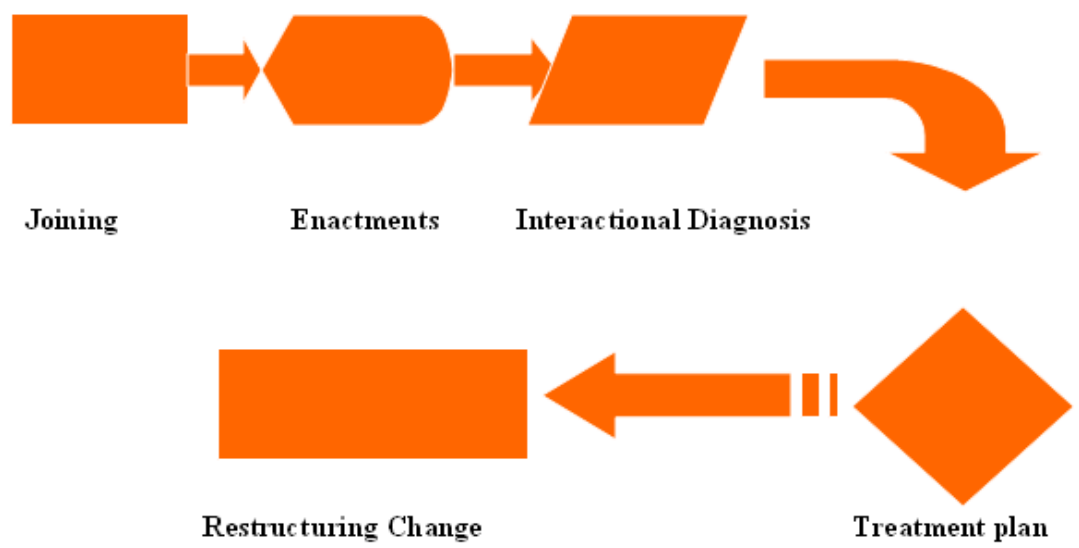

a) Establishment of a Therapeutic Relationship - Joining

Engaging adolescents and family members into treatment is usually a challenge in itself. The first step in working with a family is to establish and build a working therapeutic relationship. The construction of this relationship begins from the very first contact and that the ultimate goal of the joining process is for the therapist to form a new system - a therapeutic system, that is made up of the "whole family" and the therapist (Szapocznik, Hervis \& Schwartz, 2003). Joining the family is a strategic step which requires certain maneuvers that place the therapist in a therapeutic leadership role. In joining the therapist exercises what may be referred to as "political skills".

Joining must occur at two levels: at the individual level with each participating family member; and at the family level in which the therapist must recognize, respect, and maintain the family's characteristic interactional patterns. The desired qualities of the therapeutic relationship are respect, empathy, and commitment to working toward achieving the goals formulated between the therapist and the family. Strategies suggested to maintain the quality of the therapeutic relationship in family therapy include validating or supporting family members, formulating personally meaningful goals, and attending to each client's experience (Diamond, Hogue, Liddle, \& Dakof, 1999; Diamond \& Liddle, 1996). By demonstrating respect and acceptance of all family members, establishing a 
working alliance with each family member, and blending with the family, the therapist is finally accepted as a "special temporary member" of the family for the purpose of treatment.

The therapist needs to have an understanding of how the family is organized, and works her/his way into the family through the existing structure. In joining, the therapist should not rush to change the family system. Instead, during joining the BSFT therapist earns her/his way into the family system. Challenging the existing structure prematurely will result in a failure to engage a family into treatment. Besides affording each family member a personal experience of the therapist's regard and commitment to her/his wellbeing, it is of crucial importance that the family perceive the therapist as the leader of the therapeutic system. Families come for help with problems that they have not been able to resolve by themselves. They expect, need and are entitled to a therapist who will "lead" them in a new and more effective direction. Therefore, in the therapeutic system, the therapist is both a member and its leader. To earn this position of leadership, the therapist must offer clear rules that serve the needs of all family members, and the therapist must show respect for all family members, and in particular, for powerful family members.

\section{b) Creating Enactments}

It is necessary to have formulations to organize strategies of change. BSFT diagnostic categories organize all of the observations of a family's interactional patterns. Diagnosis of family patterns of interactions is based on what families do in the therapy sessions which the therapist observes, and not on what the family reports they do outside the session. BSFT therapists must create a therapeutic context where family members are free to interact in their typical style. These "enactments" permit the therapist to directly observe how the family behaves at home (outside of the session) and are critical for accurately identifying the family's characteristic pattern of interaction. Family crises are opportune moments in which families manifest vividly their patterns of interactions, usually highly charged and families are more emotionally available to try new behaviors. It is the process of how the family interacts that will be crucial in observing and diagnosing the interactional patterns, not the content of what the family is bringing to session that matters to the BSFT therapist. $\boldsymbol{B S F T}$ is not interested in why problems got started. It is concerned with present observable interactions, not with events that were fixed in time and are out of the reach of therapeutic intervention.

\section{c) Interactional Diagnosis}

The BSFT Diagnostic Schema will allow the therapist to focus on process, not content; organize observed behavior into patterns of interactions. Strategically identify the interactional patterns that are most amenable to change or which represent the best points of entry; formulate specific, focused treatment plans to change maladaptive patterns of interactions.

Table 1 represents maladaptive patterns of interaction observed in families of Latin American origin served in Miami who haveadolescents with behavior problems, specifically drug abusing adolescents. 


\section{Table 1. Maladaptive patterns of interaction observed} in families with adolescent behavior problems

Organization:

Resonance:

Conflict-Resolution:

Identified Patienthood:

Developmental Stage:

\section{Life Context:}

- Powerlessness in parent figures

- Unbalance in the parental or executive subsystem

- Powerful adolescents (drug user)

- Ineffective parenting

- Disrespect of parents

- Triangulated interactional sequences

- Coalitions between one parental figure and the identified patient against all other parental figure(s).

- Lack of participation of the identified patient (drug user) in the sibling subsystem.

- User (Identified patient) and his/her "protector" are enmeshed

- "Other" parent is distant, disengaged from both the user (adolescent) and the "protecting" parent.

- Close and enmeshed relationships are often very conflictive, but not truly intimate. Lots of arguments.

- No intimacy with the disengaged family members (often a spouse).

- High levels of conflict, a pattern of non-resolution.

- Inability for constructive confrontation and negotiation.

- The "drug user" or "identified patient" is very centralized by virtue of high levels of negativity or being a constant topic of conversation.

- Identified patient is viewed as the source of most of the family pain and unhappiness.

- The user is infantilized in that $\mathrm{s} / \mathrm{he}$ functions at a lower level of roles and

responsibilities within the home than expected for his/her age.

- Other siblings "pick up the slack" or "compensate for the family"

by assuming roles and responsibilities beyond their age.

- The family is typically isolated from pro-social, wellfunctioning systems.

- The family is intensely involved with other dysfunctional systems such as

gangs, and systems that control or treat dysfunctionsuch as the legal system, the mental health system and schools for troubled youth 


\section{d) Treatment Plan}

The main goal in treatment is to help the family change the maladaptive interactional patterns that are identified during the diagnostic process. The therapist plans how s/he will intervene to help the family move from its present way of interacting and the undesirable symptoms they produce, to a more adaptive and successful way of interacting that will eliminate these symptoms.

e) Restructuring Change

The interventions used to help families move from their maladaptive patterns of interactions to healthier patterns are called restructuring. Some frequently used restructuring techniques follow.

\section{Working in Present: Process vs. Content}

The distinction between process and content is absolutely critical to $\boldsymbol{B S F T}$. In her/his work, the $\boldsymbol{B S F T}$ therapist targets the family's patterns of interactions that are linked to the problem symptoms. To be able to identify these repetitive patterns of interactions, it is essential for the $\boldsymbol{B S F T}$ therapist to understand the difference between process and content. Although content is not ignored, the heart of assessment and intervention in BSFT is process focused.

Process is the "how" of what people do, and the "what happens" in an interaction. Process describes the flow of actions and reactions between family members. The repetitive actions and reactions between and among family members becomes the focus of planned interventions in BSFT. On the other hand, content refers to the specific or concrete facts used in the communication. Content includes the reasons families give for a particular interaction. Families that are unaware of their maladaptative patterns of interactions will try to engulf the therapist with historical descriptions rich in content.

Creating a context in which families behave as they would at home, the therapist is able to focus what is happening here and now, rather than getting trapped in the content (reasons the family provide or the stories they tell) of what happened there and then. This focus permits therapists to move beyond simply asking the family members to talk about behaving differently, to helping them to learn new skills and interactions during sessions.

\section{Reframing}

Reframing is the formulation of a "different" perspective or "frame" of reality than the one within which the family has been operating. The therapist presents this new frame to the family in a manner that "sells" the new frame to the family. A reframe strategically selects to present a view of reality that can facilitate change. It is important to note that in BSFT the goal of reframing is NOT to change individual cognition. Rather, the goal is to disrupt rigid, maladaptive family interaction patterns and create a new context in which family interactions can occur. Reframing is often used to disrupt negative perceptions by offering positive alternatives to the family. This shift from negative interactions to positive interactions may then lead to improvements in all other areas of family functioning.

The major goal of all restructuring interventions is to create the opportunity for the family to behave in new ways. Reframing is usually critical for reducing negativity and IP'hood in families with adolescent behavior problems. Shifting family members from viewing the IP as a "sociopath" to a "vulnerable child that is in pain" can profoundly influence family interactions. Such a shift in focus is not easy to attain. Therapists must be 
ready to offer many reframes and to present many variations of the theme. Only by consistently attending to family members' anger and the IP's pain will the therapist be able to change the characteristic pattern of interactions.

Reframing is a very safe intervention. For that reason, the $\boldsymbol{B S F T}$ therapist uses it even in the most explosive of situations. An experienced therapist is always equipped with some standard reframes that he or she can access in various situations: anger as pain or loss (underlying the anger), highly conflictive relationships as close or passionate, crises as opportunities (to pull the family closer, to become a stronger person, etc), feeling overwhelmed as a signal that one must recharge one's batteries, impulsiveness as spontaneity, and insensitivity as 'telling it as it is' (Mitrani, Szapocznik, \& RobinsonBatista, 2000).

\section{Working with Boundaries and Alliances}

Family members must form alliances to carry out important functions in the family. For example, a strong parental alliance facilitates effective behavior control and nurturance of children. Much of the work in BSFT involves helping family members establish adaptive alliances that meet the needs of the family and its individual members. This often involves disrupting harmful alliances.

The BSFT therapist addresses maladaptive alliances by reorganizing relationships within the family. This is called shifting boundaries. An alliance basically denotes the existence of a subsystem, which has boundaries around it. To change the nature of an alliance, the therapist shifts the boundaries that connect some family members together, and keep others apart. For example, rather than directly confronting an over-involved motherdaughter subsystem, the therapist may ask the father to speak directly with the daughter and/or encourage mother-father interactions to strengthen the parental alliance. As noted, this is often accomplished by blocking direct communications between the connected family members and/or by bringing a new family member into the dialogue.

The parenting subsystem is central in nearly all BSFT interventions. Building a strong leadership subsystem with clear boundaries and the power to carry out leadership functions is critical for the life of the family. In working with behavior problem adolescents, it is important that the therapist recognize that members of the parental subsystem have complex relationships that involve more than parenting functions. Issues from other aspects of the relationship often interfere with effective parenting. Although these other issues (such as marital conflict) can be addressed in treatment, BSFT is not intended to resolve all of the problems encountered by the marital couple. Because of the strategic, problem-focused nature of BSFT, the therapist tries to resolve only those aspects of their difficulties with each other that are interfering with their ability to manage the problems with their youth.

Another common alliance problem occurs when some family members are enmeshed or overly close with each other. Many times, the enmeshed subsystem is also disengaged or distant from the rest of the family. Reorganizing relationships in these instances involves facilitating appropriate levels of connection among family members. A major goal of interventions is to increase the directness and specificity of communication among all family members. By opening up lines of communication, the therapist creates more flexibility in the family; hence there are more opportunities (and less rigidity) for interaction. 
Boundary shifting also involves the clear demarcation of the perimeter around a subsystem. There are times, for example, when children interfere with the relationship between the parents. In this example, boundaries between the generations are weak and need to be more clearly marked. The therapist can make it understood that parents have a right to a certain amount of privacy (content) to achieve a clearer demarcation of limits between the generations (process). In the same way, children and adolescents have the right to a certain amount of privacy, and it is often necessary to mark the boundaries or limits of how much parents should intrude in a child's or adolescent's life.

As the therapist will learn through experience with families that have problems with boundaries, the most difficult part of the job is to get parents to stick to their side of the contract. From a BSFT point of view, it is very important that the therapist help the parent(s) develop adequate boundaries with their behavior problem youths.

\section{Assignment of Tasks}

In $\boldsymbol{B S F T}$ the use of tasks is central to all work with families. Tasks are used both inside and outside the therapy sessions as a basic tool for orchestrating change. Because emphasis is in promoting new skills among family members, both at the level of individual behaviors and family interactional relations, tasks are the vehicle through which therapists compose opportunities for the family to behave differently.

It is a general rule that the $\boldsymbol{B S F T}$ therapist must first assign a task to be performed within the session, where the therapist has an opportunity to observe, assist, and facilitate the successful conduct of the task. The therapist's aim is to create a successful experience for the family. Thus, the therapist should start with easy tasks, and work up to the more difficult ones, slowly building a foundation of successes with the family, before attempting truly difficult tasks; and move from inside the session tasks to the same task outside the session.

For failed tasks the mission will be to identify the obstacle(s) and then help the family to overcome them. Actually, failed tasks are usually a great source of new and important information about the interactions that prevent a family doing what is best for them

\section{Termination of Treatment}

Termination occurs when it is clear that the family has met the goals of the treatment plan; that is, family functioning has improved and adolescent behavior problems have been reduced or eliminated. Thus, termination is not determined by the number of sessions provided, but by the improvement in identified behavioral criteria. BSFT is designed to be delivered in 12-16 directive and active sessions, with booster sessions implemented as needed. After successful termination, families may come across some of the old or even some new problems for which they may receive booster sessions. At this point, even a troubled situation for the family is different from their experience in a prior era because each member has already enjoyed the benefits of a better functioning family. 


\section{Hard-To-Reach Families}

\section{Common Identified Problems \& Specific Strategic Approaches}

Engagement and retention in child and adolescent problem behavior treatment is difficult (Kazdin, 1994). Families with adolescent behavior problems are very difficult to engage in treatment. While one member of the family may want treatment, others do not. We have spent considerable time and effort to identify specific "family-level" obstacles to engagement and we have developed specific strategic interventions for engaging resistant families. We call these strategies BSFT -Engagement (Szapocznik, Perez-Vidal, et al., 1990; Szapocznik and Kurtines, 1989). In BSFT, resistance is viewed systemically and is understood within the context of family member's actions and reactions in relation to the therapist's behaviors. Therefore, resistance occurs when the family behaves as it naturally does, and the therapist's behavior is unable to overcome the family's status quo. Or said differently, when the therapist behaves in ways that elicits the family's typical behaviors. The solution and first goal in BSFT- Engagement is to overcome the family's usual patterns of interaction that permits the symptom of resistance to entry into treatment. The therapist must identify family interaction patterns that may interfere with engagement and then adjust their own behavior to minimize family resistance. The engagement process begins with the first call, and patterns of family interactions that are described can be rapidly identified. The overall goal at this point is to strategically bring the family into treatment. The therapist must be aware that the strategic goal at this point is engagement rather than restructuring the patterns of behaviors that impede engagement.

In fact, it is by strategically respecting and accepting (rather than challenging) the family patterns that the therapist is able to join (be accepted by) the family and its individual members. Once the family is engaged in treatment, the actual treatment of maladaptive patterns of interactions begins.

Four general patterns of family interaction that interfere with engagement into treatment have been identified. These four patterns are discussed below in terms of how the resistant patterns of interactions are manifested, and how they come to the attention of the therapist

\section{Powerful Identified Patient}

With behavior problem adolescents, the most frequently observed pattern of family interaction that interferes with engagement is a powerful identified patient (IP). This is particularly a problem in cases that are not court-referred, and therefore the adolescent IP has no reason to engage in therapy. These families are characterized by an IP who is in a powerful position in the family, and whose parents, conversely, are unable to influence the adolescent.

To bring these families into treatment, the youth's power in the family is not directly challenged, but rather it is accepted and tracked by the therapist. The therapist allies her/himself to the powerful adolescent so that s/he may later be in a position to influence the adolescent to change. This often involves some individual meetings with the adolescent either by phone or in person. Individual therapy is not the purpose of these meetings; rather the work focuses on how to bring the youth into treatment. The initial goal is to form an alliance with the powerful adolescent and to reframe the need for therapy in a manner that allows the adolescent to perceive her/himself in a powerful way and her/his position in the 
family in a positive way. The kind of reframing that is most useful with powerful adolescents involves transferring the symptom from the powerful IP to the family system. The therapist should spend a disproportionate amount of time and energy targeting the powerful member's entry into family therapy. This does not mean that therapists ignore building a relationship with parents but forming the initial alliance with the parents is likely to be ineffective because the parents are not strong enough to bring their adolescent into therapy.

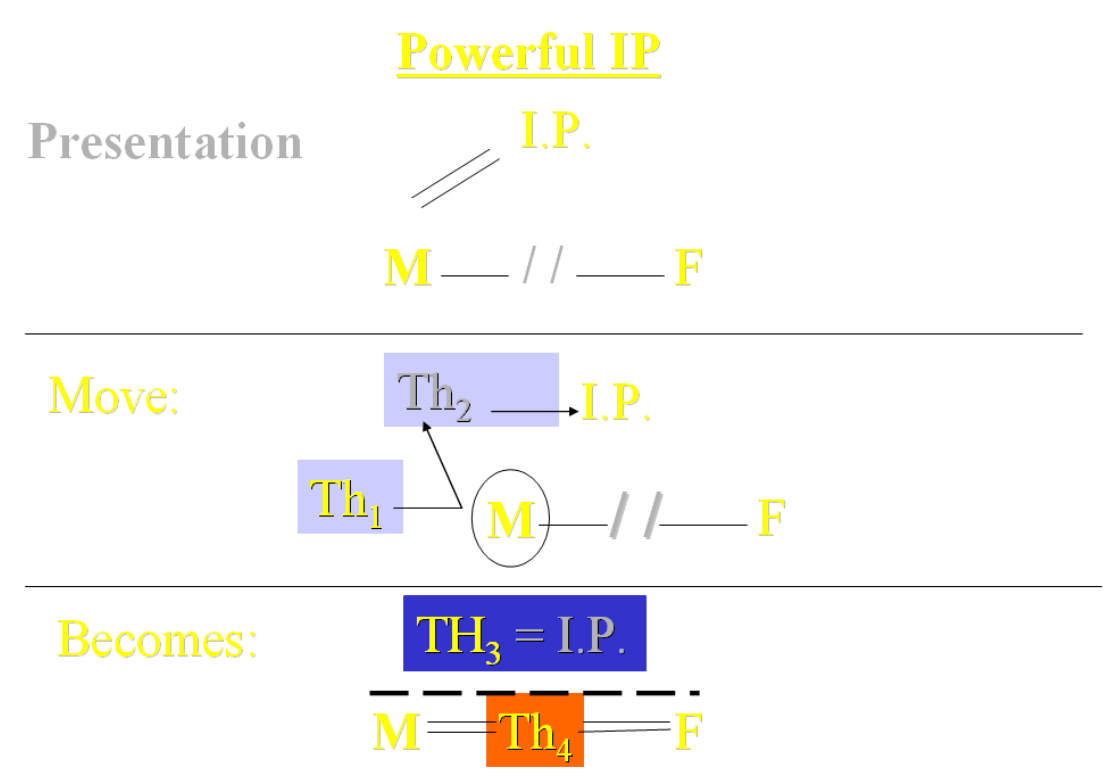

Figure 2

2. Caller Protecting the Symptom

A parent who protects the family's maladaptive patterns of interaction characterizes the second most common type of resistance to entering treatment. These families are identified when the person making the agency contact (usually the mother) to request help is also the person protecting the resistance to entering therapy. To bring these families into treatment, the therapist must first form an alliance with the mother by acknowledging her frustration in wanting to get help and not getting any cooperation from the family member(s). Through this alliance, the therapist asks the mother's permission to contact the other family members "even though they are busy" and the therapist recognizes how difficult it is for them to become involved. With the mother's permission, the therapist calls the other family members and "separates" them from the mother around the issue of coming to therapy. The therapist does this by developing her/his own relationship with other family members about the importance of coming to therapy. By the therapist developing his own relationship with these other family members, he circumvents the mother's protective 
behaviors. Once in therapy, the mother's overprotection of the adolescent's misbehavior and the mother's overprotection of the father's lack of involvement (and the adolescent and father's eagerness that mother continue to protect them) will be addressed as maladaptive patterns of interactions related to the adolescent's presenting problem behaviors.

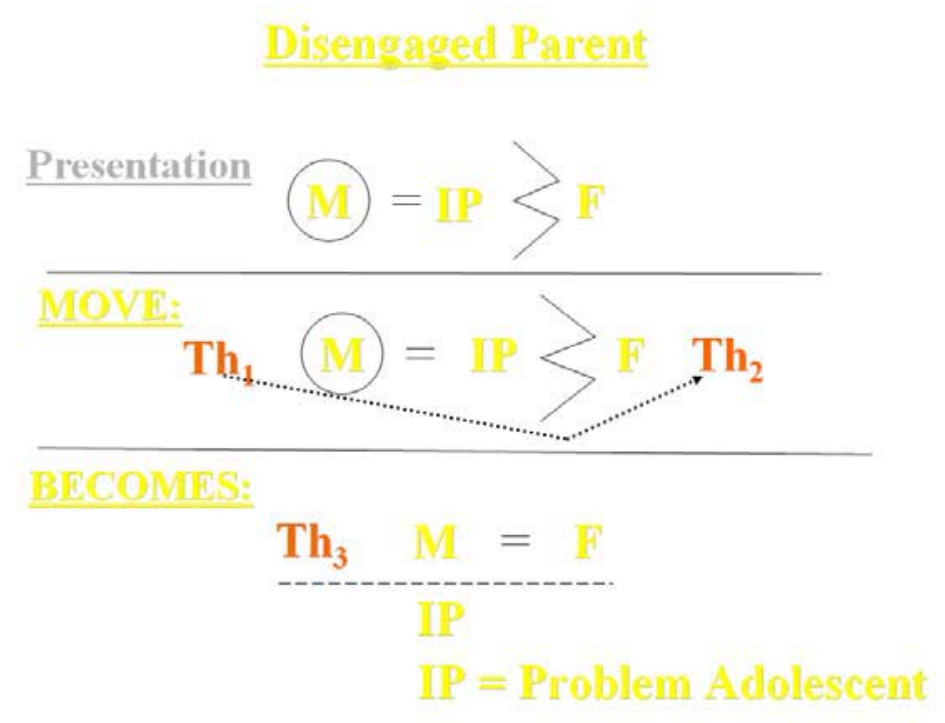

Figure 3

\section{Disengaged Parent}

These family organizations are characterized by little or no cohesiveness or alliance between the parents or parent figures as a subsystem. One of the parents, usually the father, refuses to come into therapy. This is typically a parent/father who has remained disengaged from the problems at home. Parent disengagement not only protects the parent from having to address adolescent behavior problems, but it also often protects the parents from having to deal with aspects of their own (marital) relationship. In many circumstances, the engaged parent is over-involved (enmeshed) with the IP and may be inadvertently supporting the adolescent's problem behaviors.

To engage these families into treatment, the therapist must form an alliance with the caller (usually the mother). The therapist then begins to direct the mother's interactions with the father, changing their patterns of interaction so as to improve, at least temporarily, their cooperation in bringing the family into treatment. The therapist gives mother tasks to do together with her husband pertaining only to the issue of taking care of their son's problems by getting the family into treatment. The therapist assigns tasks and coaches the mother to act in such a way that is least likely to spark the broader marital conflict. These tasks are intended to restructure the marital couple's interaction only around the whole family coming to treatment. To set up the task, the mother may be asked what she believes is the "real" reason her husband does not want to come in. Once this is ascertained, she is 
coached to present coming to treatment in a way that the husband can accept. Although the pattern is similar to that of the contact person protecting the organization, in this instance the resistance emerges differently. In this case the mother does not excuse the father's distance but, quite the contrary, she complains about her spouse's disinterest; this mother is usually eager to do something to involve her husband, given some direction.

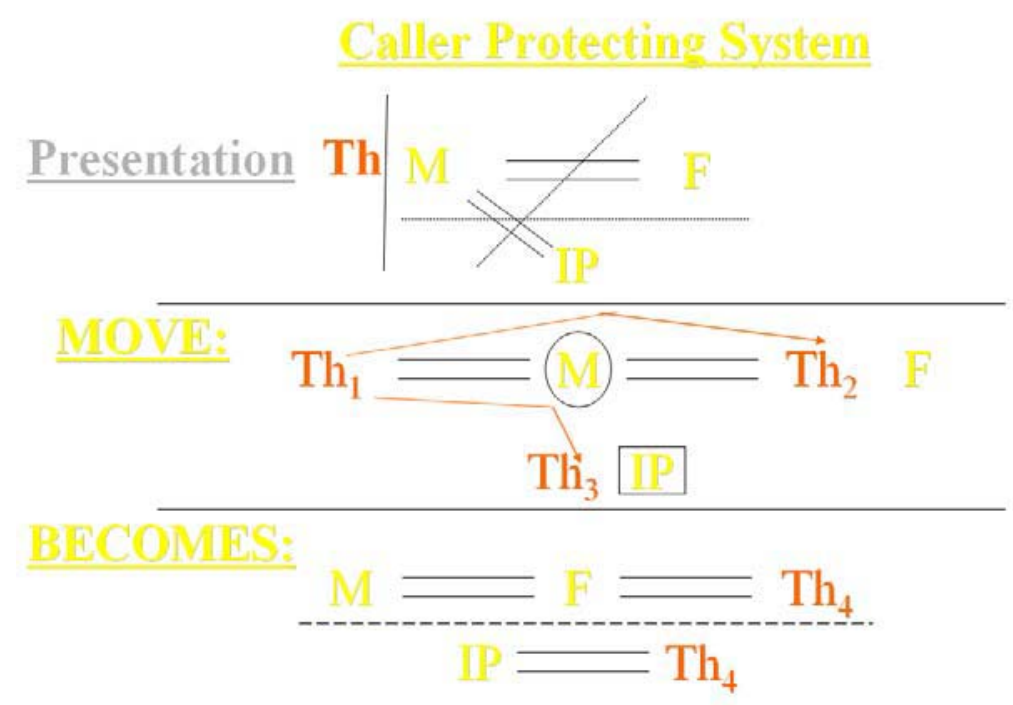

Figure 4

4. Fear of Therapy as an Expose

Therapy might be a threatening idea to one or more individuals. Sometimes the person who resists coming is afraid of being made a scapegoat or that dangerous secrets will be revealed These individuals' beliefs or "frames" about therapy are usually an extension of the "frame" within which the family is functioning.

The therapist must reframe the idea or goal of therapy in a way that eliminates its negative consequences and replaces them with positive aims. One example is to meet with the person who is most rejecting of therapy and assure him that therapy does not have to go where s/he does not want it to go. The therapist will make every effort to focus on the problems of the youth, and will not focus on the issues that might concern the unwilling family member. The individual is also assured that in the session "we will deal only with those issues that you want to deal with." In our experience, however, even if the therapist focuses on those aspects of the marital/parenting relationship that are directly related to the youth, sometimes the frightening secrets will be revealed in therapy; and their revelation is likely to have a positive long term effect. 
Therapists should keep in mind that many other family patterns likely may interfere with engagement. The important frame is that therapists pay attention to identifying family patterns from the first contact and that $s$ /he adjust their engagement strategy to maximize their chances for bring family members to treatment. Also, therapists should note that adjusting strategies does not mean restructuring family interactions, but rather refers to respecting the family's organization and strategically connecting with appropriate members of the family. It is important to note, that even if the above identified problems call for one to one interactions between the BSFT therapist and a member of the family, BSFT by no means intends to address individual problems. Individual contacts are rather used as a bridge to the family, when engagement to treatment is compromised.

\section{Research Evidence}

Outcome research findings for BSFT are briefly presented below, including the impact of BSFT on: 1) adolescent drug use, 2) engagement and retention, 3) behavior problems, and 4) family functioning.

\section{Engagement and Retention}

In three separate studies (Coatsworth, Santisteban, McBride, \& Szapocznik, 2001; Santisteban et al., 1996; Szapocznik et al., 1988) the efficacy of specialized BSFT engagement strategies in engaging and retaining drug abusing adolescents and their families in treatment have been demonstrated. Szapocznik, Perez-Vidal et al. (1988) demonstrated that $93 \%$ of families that received the BSFT-Specialized Engagement were successfully engaged, as compared to $42 \%$ that did not receive the specialized engagement intervention. Moreover, the utilization of the same specialized engagement strategies to retain cases in treatment resulted in $77 \%$ of families in the Specialized Engagement condition receiving a full dose of therapy (approximately 8 sessions), compared to $25 \%$ of families that did not receive these interventions. Not only did the addition of the BSFT specialized engagement module triple retention in treatment, but those who were retained in both conditions (three times as many with specialized engagement) showed significant improvements in drug use, conduct problems and family functioning. Although BSFT specialized engagement brought into treatment more difficult to engage families, once in treatment they benefited from BSFT treatment like any other family. These findings were replicated by Coatsworth, Santisteban, McBride, \& Szapocznik, 2001 and Santisteban et al., 1996 in two different clinical randomized trials testing the power of BSFT specialized engagement.

\section{Child/Adolescent Treatment Outcomes}

In one study, BSFT was compared against Individual Child Psychodynamic Psychotherapy and recreation control group. Sixty-nine Hispanic moderately emotionally or behaviorally troubled 6 to 11 year old boys were randomly assigned to one of the three intervention conditions (Szapocznik, Rio et al., 1989).

The results of the analyses revealed several important findings. The first finding indicates that the control condition (i.e., recreation activities) was significantly less effective at retaining cases than the two treatment conditions, with over two thirds of all dropouts occurring in the control condition. The two treatment conditions had equivalent rates of retention, thus differences in treatment outcome between the treatment groups were 
most likely due to the treatment interventions. The second finding was that the two treatment conditions, $\boldsymbol{B S F T}$ and child psychodynamic, equivalently reduced emotional and behavior problems (parent and child reports) and child psychodynamic functioning. The third and most significant differential treatment finding was that family therapy was more effective than child therapy at protecting family integrity at the one-year follow-up. Although individual psychodynamic child therapy was found to be efficacious at reducing behavior and emotional problems as well as improving child psychodynamic functioning, it was also found bring about deterioration of family functioning at the one-year follow up. In contrast, the family therapy condition brought about significant improvement of family functioning at the one-year follow up. The findings provided support for the $\boldsymbol{B S F T}$ assumption that treating the whole family is important because it improves the symptoms and protects the family.

BSFT was also compared to group counseling (Santisteban et al., 2003; in a randomized trial. They found that for adolescents who abused marijuana, $75 \%$ showed reliable improvement, $56 \%$ were classified as recovered, and $25 \%$ showed reliable deterioration in the BSFT condition. In contast, $14 \%$ showed reliable improvement and were classified as recovered, and $43 \%$ showed reliable deterioration in adolescent group counseling. BSFT was far better in bringing about improvements in marijuana use. In contrast, group counseling was much more likely to cause the adolescents to worsen in their marijuana use.

In another outcome variable for the same study, results on externalizing behaviors (conduct problems and delinquency in the company of peers)showed that adolescents in BSFT improved dramatically more (statistically significantly) when compared with adolescents in group counseling adolescents. Specifically, an analyses of clinical significance in conduct disorder showed that in the BSFT condition $44 \%$ showed reliable improvement, $26 \%$ were classified as recovered, and $5 \%$ showed reliable deterioration. In the Group Control condition $11 \%$ showed reliable deterioration and no case was classified as reliably improved or recovered. A similar pattern was seen for delinquency in the company of peers. Thus, in the study in all there adolescent outcomes BSFT Was dramatically better than group counseling.

\section{Family Functioning}

BSFT theory is based on the assumption that the family is considered to play a critical role in the development, maintenance, and treatment of adolescent behavior problems. Studies examining the impact of BSFT on family interactions (Szapocznik et al., 1983) have shown significant impact of BSFT in family functioning (as measured among the five diagnostic dimensions described in section III). The most recent study demonstrated that when compared to a group counseling, BSFT produced increases in adolescent-reported family cohesion and improvements in observer-reported family functioning. Group counseling in contract produced deterioration in family functioning.

\section{The Clinical Interior of BSFT}

Two studies have been conducted to examine aspects of the clinical interior of BSFT. The first study demonstrated that improvements in family interactions as observed in therapy sessions was associated with improvements in behavioral outcomes (Robbins et al., 2000). This study is particularly important because it demonstrated the link between improvements in structural interaction patterns and clinical outcome in BSFT. A second 
study demonstrated that unbalanced alliances with the therapist in early sessions of BSFT was associated with dropout (Robbins et al., 2004). Specifically, this study showed that families that dropped out of treatment were characterized by larger discrepancies in mothertherapist and adolescent-therapist alliances than families that completed BSFT. Also, the father-therapist alliance was significantly higher in families that completed compared to families that dropped out of treatment. These alliance findings suggest that family patterns of alliance are particularly important in understanding the effectiveness of BSFT. Moreover, these findings indicate that alliance with fathers also plays a critical role in understanding the effects of treatment. Because both of these studies included Hispanic families only, these results may not generalize to other interventions and cultures.

\section{Future Research Challenges}

Currently, a research study is being conducted to compare the effectiveness of BSFT to treatment as usual for adolescent drug users. This study is being implemented in 14 community treatments programs throughout the country. This study is part of national collaboration of 17 university centers under the auspices of the Clinical Trials Network of the National Institutes for Drug Abuse. The present study will randomized 840 adolescents and their families across the 14 sites to either BSFT or the agencies usual treatment for these adolescents. Thus, BSFT will be compared to treatment in the real world. The research study will also examine how BSFT compares to treatment as usual in reducing sexually risky behaviors, in reducing externalizing behaviors, in improving family functions and in promoting pro social activities. As part of a joint collaboration with researchers at the University of Arizona, the study will also examine how and for whom $\boldsymbol{B S F T}$ works. This study is being conducted by therapists in these community agencies. The therapist who will be conducting the study at the community agencies around the country have been trained and are supervised throughout the study by BSFT Trainers from the Center for Family Studies in Miami, Florida, U.S.A. José Szapocznik is the Lead Investigator of this national study. Michael Robbins is the co-Lead Investigator and Project Director. Viviana Horigian is the National Protocol Coordinator.

This work is funded by National Institute on Drug Abuse Grant \# DA 13720 to Professor José Szapocznik, Principal Investigator

\section{References}

Coatsworth, J.D., Santisteban, D.A., McBride, C.K., \& Szapocznik, J. (2001). Brief Strategic Family Therapy versus community control: Engagement, retention, and an exploration of the moderating role of adolescent symptom severity. Family Process, 40 (3), 313-332.

Diamond, G. \& Liddle, H.A. (1996). Resolving a therapeutic impasse between parents and adolescents in multidimensional family therapy. Journal of Consulting and Clinical Psychology, 64(3), 481-488.

Diamond, G.M., Liddle, H.A., Hogue, A., \& Dakof, G.A. (1999). Alliance-building interventions with adolescents in family therapy: A process study. Psychotherapy: Theory, Research, Practice, Training, 36(4), 335-368.

Kazdin A.E. (1994). Interventions for aggressive and antisocial children. In L.D. Eron \& J.H.

Gentry's (Eds.) Reason to hope: A psychosocial perspective on violence and youth. Washington, DC: American Psychological Association, pp. 341-382. 
Mitrani, V.B., Szapocznik, J., \& Robinson Batista, C. (2000). Structural ecosystems therapy with HIV+ African American women. In W. Pequegnat \& J. Szapocznik (Eds.) Working with families in the era of HIV/AIDS (pp. 3-26). Thousand Oaks, CA: Sage.

Robbins, M.S., Alexander, J.F., \& Turner, C.W. (2000). Disrupting defensive family interactions in family therapy with delinquent adolescents. Journal of Family Psychology, 14(4), pp. 688-701.

Robbins, M.S., Turner, CW., Mayorga, C.C., Alexander, J.F., \& Szapocznik, J. (manuscript in preparation). Adolescent and Parent Alliances with Therapists in Brief Strategic Family Therapy with Behavior Problem Hispanic Adolescents.

Santisteban, D.A., Coatsworth, J.D., Perez-Vidal, A., Kurtines, W.M., Schwartz, S.J., LaPerriere, A., Szapocznik, J. (2003). Efficacy of brief strategic family therapy in modifying Hispanic adolescent behavior problems and substance use. Journal of Family Psychology, 17(1), 121-133. Santisteban, D.A., Szapocznik, J., Perez-Vidal, A., Kurtines, W.M., Murray, E.J. \& LaPerriere, A. (1996). Efficacy of intervention for engaging youth and their families into treatment and some variables that may contribute differential effectiveness. Journal of Family Psychology, 10(1), 35-44.

Szapocznik, J. \& Coatsworth, J.D. (1999). An ecodevelopmental framework for organizing the influences on drug abuse: A developmental model of risk and protection. In M.D. Glantz \& C.R. Hartel's (Eds.) Drug abuse: Origins \& interventions. Washington, DC: American Psychological Association, pp. 331366.

Szapocznik, J., Hervis, O.E., with Schwartz, S. (2003). Brief Strategic Family Therapy manual [NIDA Therapy Manuals for Drug Addiction Series]. Rockville, Maryland: National Institute on Drug Abuse.

Szapocznik, J. \& Kurtines, W. (1989). Breakthroughs in family therapy with drug abusing problem youth. New York: Springer.

Szapocznik, J., Kurtines, W.M., Foote, F. \& Perez-Vidal, A. (1983). Conjoint versus one-person family therapy: Some evidence for effectiveness of conducting family therapy through one person. Journal of Consulting and Clinical Psychology, 51, 889-899. Indexed in Inventory of Marriage and Family Literature (IMFL), 1985, Vol. XI. Beverly Hills, California: Sage Publications.

Szapocznik, J., Perez-Vidal, A., Brickman, A.L., Foote, F.H., Santisteban, D., Hervis, O. \& Kurtines, W.M. (1988). Engaging adolescent drug abusers and their families in treatment: A strategic structural systems approach. Journal of Consulting \& Clinical Psychology, 56, 552-557.

Szapocznik, J., Perez-Vidal A., Hervis, O.E., Brickman, A.L. \& Kurtines, W.M. (1990). Innovations in family therapy: Strategies for overcoming resistance to treatment. In R.A. Wells \& V.J. Giannetti (Eds.) Handbook of the brief psychotherapies (pp. 93-114). New York: Plenum.

Szapocznik, J., Rio, A., Murray, E., Cohen, R. (1989). Structural family versus psychodynamic child therapy for problematic Hispanic boys. Journal-of-Consulting-and-Clinical-Psychology, 57(5), 571-578.

\author{
Address reprint requests to: \\ José Szapocznik \\ Department of Psychiatry and Behavioral Sciences \\ University of Miami School of Medicine \\ 1425 , N. W. $10^{\text {th }}$ Avenue, No. 207 \\ Miami, Florida, 33136, USA
}

